\title{
MULTIPLE SOLUTIONS OF PERIODIC BOUNDARY VALUE PROBLEMS FOR FIRST-ORDER DIFFERENCE EQUATIONS
}

\author{
DA-BIN WANG
}

\section{(Received 4 June 2007)}

\begin{abstract}
In this paper, existence criteria for multiple solutions of periodic boundary value problems for the firstorder difference equation

$$
\left\{\begin{array}{l}
\triangle x(k)=f(k, x(k+1)), \quad k \in[0, T], \\
x(0)=x(T+1),
\end{array}\right.
$$

are established by using the Leggett-Williams multiple fixed point theorem and fixed point theorem of cone expansion and compression. Two examples are also given to illustrate the main results.
\end{abstract}

2000 Mathematics subject classification: 39A10.

Keywords and phrases: periodic boundary value problem, positive solution, fixed point theorem, difference equation, cone.

\section{Introduction}

Due to its wide application in many fields such as science, economics, neural networks, ecology and cybernetics, the theory of nonlinear difference equations has been widely studied since the 1970s; see, for example, [1, 6, 21, 22]. At the same time, boundary value problems (BVPs) of difference equations have received much attention from many authors; see [2-5, 7-13, 15, 18-20, 25-31] and the references therein. However, to the best of our knowledge, few papers can be found in the literature for periodic boundary value problems (PBVPs) of difference equations [9, 10, 25, 27].

In this paper, we are concerned with the existence of multiple solutions of the PBVP for the first-order difference equation

$$
\left\{\begin{array}{l}
\triangle x(k)=f(k, x(k+1)), \quad k \in[0, T], \\
x(0)=x(T+1),
\end{array}\right.
$$

(c) 2008 Australian Mathematical Society 0004-9727/08 \$A2.00+0.00 
where $T$ is a fixed positive integer, $\triangle$ denotes the forward difference operator with stepsize $1,[a, b]=\{a, a+1, \ldots, b-1, b\} \subset Z$ (the set of all integers), and $f:[0, T] \times R \rightarrow R$ is continuous.

In [25], by using a fixed point theorem [14], Sun considered the existence of one positive solutions of the PBVP (1.1).

In this paper, by using the Leggett-Williams multiple fixed point theorem and fixed point theorem of cone expansion and compression, we investigate the existence of multiple positive solutions of (1.1). It is worth noting that the Leggett-Williams multiple fixed point theorem is used extensively in yielding three solutions for BVPs of differential or difference equations; see $[12,16,17,24,26]$ and references therein.

\section{Preliminaries}

In this section, we provide some background material from the theory of cones in Banach spaces and we then state the Leggett-Williams multiple fixed point theorem and fixed point theorem of cone expansion and compression.

Definition 2.1. Let $E$ be a real Banach space. A nonempty, closed, convex set $P \subset E$ is said to be a cone provided the following conditions are satisfied:

(i) if $x \in P$ and $\lambda \geq 0$, then $\lambda x \in P$;

(ii) if $x \in P$ and $-x \in P$, then $x=0$.

Every cone $P \subset E$ induces an ordering in $E$ given by

$$
x \leq y \quad \text { if and only if } y-x \in P .
$$

DEFINITION 2.2. A map $\alpha$ is said to be a nonnegative, continuous, concave functional on a cone $P$ of a real Banach space $E$ if

$$
\alpha: P \rightarrow[0, \infty)
$$

is continuous and

$$
\alpha(t x+(1-t) y) \geq t \alpha(x)+(1-t) \alpha(y),
$$

for all $x, y \in P$ and $t \in[0,1]$.

Let $E$ be a real Banach space with norm $\|\cdot\|$ and $P \subset E$ be a cone of $E$. We define

$$
P_{r}=\{x \in P \mid\|x\|<r\},
$$

where $r>0$.

Let $\alpha$ be a nonnegative continuous and concave functional defined on $P$. We define

$$
P(\alpha, a, b)=\{x \in P \mid \alpha(x) \geq a,\|x\| \leq b\},
$$

where $0<a<b$.

To prove our main results, we need the following Leggett-Williams multiple fixed point theorem and fixed point theorem of cone expansion and compression. 
THEOREM 2.1 ([23] Leggett-Williams fixed point theorem). Let the operator $\Phi: \overline{P_{c}} \rightarrow P_{c}$ be completely continuous and let $\alpha$ be a nonnegative continuous concave functional on $P$ such that $\alpha(x) \leq\|x\|$ for every $x \in \overline{P_{c}}$. Suppose that there exist $0<d<a<b \leq c$ such that:

$\left(\mathrm{C}_{1}\right) \quad\{x \mid x \in P(\alpha, a, b), \alpha(x)>a\} \neq \phi$ and $\alpha(\Phi x)>$ a for each $x \in P(\alpha, a, b)$;

$\left(\mathrm{C}_{2}\right) \quad\|\Phi x\|<d$ for $x \in \overline{P_{d}}$;

$\left(\mathrm{C}_{3}\right) \quad \alpha(\Phi x)>$ a for $x \in P(\alpha, a, c)$ with $\|\Phi x\|>b$.

Then $\Phi$ has at least three fixed points, $x_{1}, x_{2}$, and $x_{3}$ in $\overline{P_{c}}$ satisfying

$$
\left\|x_{1}\right\|<d, \quad a<\alpha\left(x_{2}\right), \quad\left\|x_{3}\right\|>d \quad \text { and } \quad \alpha\left(x_{3}\right)<a .
$$

THEOREM 2.2 ([14] Fixed point theorem of cone expansion and compression). Let $P$ be a cone of real Banach space $E$ and $P_{r, s}=\{x \in P \mid r \leq\|x\| \leq s\}$ with $0<r<s$. Suppose that $\Phi: P_{r, s} \rightarrow P$ is a completely continuous mapping such that one of the following two conditions is satisfied:

(i) $\|\Phi x\| \leq\|x\|$ for $x \in P,\|x\|=r$ and $\|\Phi x\| \geq\|x\|$ for $x \in P,\|x\|=s$;

(ii) $\|\Phi x\| \geq\|x\|$ for $x \in P,\|x\|=r$ and $\|\Phi x\| \leq\|x\|$ for $x \in P,\|x\|=s$.

Then $\Phi$ has a fixed point $x \in P$ such that $r \leq\|x\| \leq s$.

\section{Main results}

In this section, by defining an appropriate Banach space and cones, we impose the conditions on $f$ which allow us to apply the Leggett-Williams multiple fixed point theorem and fixed point theorem of cone expansion and compression to establish the existence of multiple positive solutions of the PBVP (1.1).

For convenience, let us list the following assumptions.

$\left(\mathrm{H}_{1}\right) \quad$ There exists a positive number $M>1$ such that

$$
(M-1) x+f(k, x) \geq 0 \text { for } k \in[0, T], x \in[0, \infty) .
$$

$\left(\mathrm{H}_{2}\right) \varlimsup_{\lim _{x \rightarrow+\infty}}((f(k, x)) / x)<0$ uniformly with respect to $k \in[0, T]$.

$\left(\mathrm{H}_{3}\right) \quad$ There exist two positive numbers $a, b$ with $b \geq a M^{(T+1)}$ and $g:[0, T] \rightarrow R$ such that

$$
f(k, x) \geq g(k) \geq 0 \quad \text { for } k \in[0, T], x \in[a, b] \text { and } \sum_{k=0}^{T} g(k)>0 .
$$

( $\left.\mathrm{H}_{4}\right) \varlimsup_{\lim _{x \rightarrow 0}}((f(k, x)) / x)<0$ uniformly with respect to $k \in[0, T]$.

Let $C=\{x \mid[0, T] \rightarrow R\}$. For $\sigma \in C$, we first consider the following linear PBVP:

$$
\left\{\begin{array}{l}
\triangle x(k)+(M-1) x(k+1)=\sigma(k), \quad k \in[0, T] \\
x(0)=x(T+1)
\end{array}\right.
$$

where $M>1$ is a constant. 
Let

$$
G(k, s)= \begin{cases}\frac{M^{-(k-s)}}{1-M^{-(T+1)}}, & 0 \leq s \leq k-1, \\ \frac{M^{-(T+1+k-s)}}{1-M^{-(T+1)}}, & k \leq s \leq T .\end{cases}
$$

Then

$$
\frac{M^{-(T+1)}}{1-M^{-(T+1)}} \leq G(k, s) \leq \frac{1}{1-M^{-(T+1)}}, \quad(k, s) \in[0, T+1] \times[0, T] .
$$

It is easy to see that the following lemma holds.

LemMa 3.1. Suppose that $M>1$. Then for any $\sigma \in C$, the PBVP (3.1) has a unique solution:

$$
x(k)=\sum_{s=0}^{T} G(k, s) \sigma(s), \quad k \in[0, T+1]
$$

In addition, if we choose $\sigma(k) \equiv 1$, then we know that

$$
\sum_{s=0}^{T} G(k, s)=\frac{1}{M-1}
$$

Let $E=\{x \mid[0, T+1] \rightarrow R\}$ be equipped with the norm $\|x\|=\max _{k \in[0, T+1]}|x(k)|$; then $E$ is a Banach space.

Now for $u \in E$, we consider the following PBVP:

$$
\left\{\begin{array}{rl}
\triangle x(k) & +(M-1) x(k+1)=(M-1) u(k+1) \\
& +f(k, u(k+1)),
\end{array} \quad k \in[0, T],\right.
$$

It follows from Lemma 3.1 that PBVP (3.5) has a unique solution

$$
x(k)=\sum_{s=0}^{T} G(k, s)[(M-1) u(s+1)+f(s, u(s+1))], \quad k \in[0, T+1] .
$$

Define an operator $\Phi: E \rightarrow E$ by

$$
(\Phi x)(k)=\sum_{s=0}^{T} G(k, s)[(M-1) x(s+1)+f(s, x(s+1))], \quad k \in[0, T+1] .
$$

It is obvious that fixed points of $\Phi$ are solutions of the PBVP (1.1). 
TheOREM 3.1. Suppose that $\left(\mathrm{H}_{1}\right)-\left(\mathrm{H}_{4}\right)$ hold. Then the PBVP (1.1) has at least three nonnegative solutions.

Proof. We define a cone $P$ in $E$ as follows:

$$
P=\{x \in E \mid x(k) \geq 0, k \in[0, T+1]\} .
$$

Then, by $\left(\mathrm{H}_{1}\right)$ it is easy to see that $\Phi: P \rightarrow P$ is completely continuous.

Define a functional $\alpha(x)$ on $P$ by

$$
\alpha(x)=\min _{k \in[0, T+1]} x(k), \quad x \in P .
$$

Obviously, $\alpha: P \rightarrow[0,+\infty)$ is nonnegative continuous and concave. Moreover, $\alpha(x) \leq\|x\|$ for each $x \in P$.

Now we are in position to certify that the assumptions of Theorem 2.1 are satisfied.

We first assert that there exists a positive number $c$ with $c \geq b$ such that $\Phi: \overline{P_{c}} \rightarrow P_{c}$.

By $\left(\mathrm{H}_{2}\right)$ we know that there exist $\varepsilon \in(0, M-1)$ and $L>0$ such that

$$
\frac{f(k, x)}{x}<-\varepsilon \quad \text { for } k \in[0, T], x \geq L .
$$

Notice that $f(k, x)$ is continuous on $[0, T] \times[0,+\infty)$, and there exists $L_{1}>0$ such that

$$
f(k, x)<-\varepsilon x+L_{1} \quad \text { for } k \in[0, T], x \in[0,+\infty) .
$$

Choose $c=\max \left\{b,\left(L_{1} / \varepsilon\right)+1\right\}$, for each $x \in \overline{P_{c}}$. In view of (3.4),

$$
\begin{aligned}
(\Phi x)(k) & =\sum_{s=0}^{T} G(k, s)[(M-1) x(s+1)+f(s, x(s+1))] \\
& \leq \sum_{s=0}^{T} G(k, s)\left[(M-1-\varepsilon) x(s+1)+L_{1}\right] \\
& \leq\left[(M-1-\varepsilon) c+L_{1}\right] \sum_{s=0}^{T} G(k, s) \\
& =\frac{1}{M-1}\left[(M-1-\varepsilon) c+L_{1}\right] \\
& <c .
\end{aligned}
$$

Thus, $\|\Phi x\| \leq c, \Phi: \overline{P_{c}} \rightarrow P_{c}$.

Secondly, we assert that $\{x \mid x \in P(\alpha, a, b), \alpha(x)>a\} \neq \phi$ and $\alpha(\Phi x)>a$ for each $x \in P(\alpha, a, b)$. In fact, the constant function

$$
((a+b) / 2) \in\{x \mid x \in P(\alpha, a, b), \alpha(x)>a\} .
$$


Moreover, for $x \in P(\alpha, a, b)$,

$$
a \leq \alpha(x) \leq x(k) \leq\|x\| \leq b .
$$

Thus, in view of $\left(\mathrm{H}_{3}\right)$ and (3.4), we see that

$$
\begin{aligned}
(\Phi x)(k) & =\sum_{s=0}^{T} G(k, s)[(M-1) x(s+1)+f(s, x(s+1))] \\
& \geq \sum_{s=0}^{T} G(k, s)[(M-1) a+g(s)] \\
& \geq(M-1) a \sum_{s=0}^{T} G(k, s)+\sum_{s=0}^{T} G(k, s) g(s) \\
& >a,
\end{aligned}
$$

as required.

Thirdly, we assert that $\|\Phi x\|<d$ for $x \in \overline{P_{d}}$. Indeed, By $\left(\mathrm{H}_{4}\right)$, there exists $\varepsilon^{\prime} \in(0, M-1)$ and $d \in(0, a)$ such that

$$
f(k, x)<-\varepsilon^{\prime} x \quad \text { for } k \in[0, T], x \in[0, d] .
$$

So, for each $x \in \overline{P_{d}}$,

$$
\begin{aligned}
(\Phi x)(k) & =\sum_{s=0}^{T} G(k, s)[(M-1) x(s+1)+f(s, x(s+1))] \\
& \leq \sum_{s=0}^{T} G(k, s)\left[(M-1) x(s+1)-\varepsilon^{\prime} x(s+1)\right] \\
& \leq\left(M-1-\varepsilon^{\prime}\right) d \sum_{s=0}^{T} G(k, s) \\
& <d .
\end{aligned}
$$

Hence, $\|\Phi x\|<d$ for $x \in \overline{P_{d}}$.

Finally, we assert that $\alpha(\Phi x)>a$ for $x \in P(\alpha, a, c)$ with $\|\Phi x\|>b$. To see this, suppose that $x \in P(\alpha, a, c)$ with $\|\Phi x\|>b$. By $\left(\mathrm{H}_{3}\right)$ and (3.2) we obtain

$$
\begin{aligned}
\alpha(\Phi x) & =\min _{k \in[0, T+1]} \sum_{s=0}^{T} G(k, s)[(M-1) x(s+1)+f(s, x(s+1))] \\
& \geq \frac{M^{-(T+1)}}{1-M^{-(T+1)}} \sum_{s=0}^{T}[(M-1) x(s+1)+f(s, x(s+1))],
\end{aligned}
$$

for $k \in[0, T+1]$. Thus, 


$$
\begin{aligned}
\|\Phi x\| & =\max _{k \in[0, T+1]} \sum_{s=0}^{T} G(k, s)[(M-1) x(s+1)+f(s, x(s+1))] \\
& \leq \frac{1}{1-M^{-(T+1)}} \sum_{s=0}^{T}[(M-1) x(s+1)+f(s, x(s+1))] \\
& =M^{(T+1)} \frac{M^{-(T+1)}}{1-M^{-(T+1)}} \sum_{s=0}^{T}[(M-1) x(s+1)+f(s, x(s+1))] \\
& \leq M^{(T+1)} \alpha(\Phi x) .
\end{aligned}
$$

That is,

$$
\alpha(\Phi x) \geq M^{-(T+1)}\|\Phi x\|>M^{-(T+1)} b \geq a .
$$

To sum up, all the hypotheses of Theorem 2.1 are satisfied. Hence, $\Phi$ has at least three fixed points, that is, (1.1) has at least three nonnegative solutions $x_{1}, x_{2}, x_{3}$ such that

$$
\left\|x_{1}\right\|<d, \quad a<\min _{k \in[0, T+1]} x_{2}, \quad\left\|x_{3}\right\|>d \quad \text { and } \min _{k \in[0, T+1]} x_{3}<a
$$

TheOREM 3.2. Assume that $\left(\mathrm{H}_{1}\right),\left(\mathrm{H}_{2}\right)$ and $\left(\mathrm{H}_{4}\right)$ hold. Assume also that there exists a positive number e satisfying $M^{(T+1)} d<e<c$ such that

$$
f(k, x)>(M-1)(1-\delta) e, \quad k \in[0, T], x \in[\delta e, e],
$$

where $\delta=M^{-(T+1)}$, then the PBVP (1.1) has at least three nonnegative solutions.

Proof. Define a cone $P$ in $E$ as follows:

$$
P=\{x \in E \mid x(k) \geq \delta\|x\|, k \in[0, T+1]\} .
$$

Then, by $\left(\mathrm{H}_{1}\right)$ and (3.2), it is easy to see that $\Phi: P \rightarrow P$ is completely continuous.

Firstly, by $\left(\mathrm{H}_{4}\right)$ and (3.7), we have that $\Phi: \overline{P_{d}} \rightarrow P_{d} \subset \overline{P_{d}}$. Then, it follows from the Schauder fixed point theorem that (1.1) has one nonnegative solution $x_{1} \in \overline{P_{d}}$. This, together with (3.7), guarantees that $x_{1} \in P_{d}$.

Secondly, from (3.6) and (3.7), it is easy to see that

$$
\|\Phi x\|<\|x\| \quad \text { if }\|x\|=c ; \quad\|\Phi x\|<\|x\| \quad \text { if }\|x\|=d .
$$

Finally, by definition of $P$, for each $x \in \partial P_{e}=\{x \in P \mid\|x\|=e\}$, we know that $\delta e \leq x(k) \leq e, k \in[0, T]$. 
Combining this with hypothesis of theorem, we obtain

$$
\begin{aligned}
(\Phi x)(k) & =\sum_{s=0}^{T} G(k, s)[(M-1) x(s+1)+f(s, x(s+1))] \\
& >\sum_{s=0}^{T} G(k, s)[(M-1) \delta e+(M-1)(1-\delta) e] \\
& =(M-1) e \sum_{s=0}^{T} G(k, s) \\
& =e .
\end{aligned}
$$

From this and (3.8), applying Theorem 2.2 twice, it follows that there exist two positive solutions $x_{2}$ and $x_{3}$ satisfying $x_{2} \in P_{e} \backslash \overline{P_{d}}$ and $x_{3} \in P_{c} \backslash \overline{P_{e}}$.

When $f$ is superlinear at $\infty$, we have the following results.

THEOREM 3.3. Suppose that $\left(\mathrm{H}_{1}\right)$ and:

( $\left.\mathrm{H}_{5}\right) \quad \underline{\lim }_{x \rightarrow 0+}((f(k, x)) / x)>N$ uniformly with respect to $k \in[0, T]$, where $N=(((M-1)(1-\delta)) / \delta)$;

$\left(\mathrm{H}_{6}\right) \quad$ there exist e and $h:[0, T] \rightarrow R$ such that

$$
f(k, x) \leq h(k) \leq 0 \quad \text { for } k \in[0, T], \quad x \in[\delta e, e] \quad \text { and } \quad \sum_{k=0}^{T} h(k)<0
$$

( $\left.\mathrm{H}_{7}\right) \underline{\lim }_{x \rightarrow+\infty}((f(k, x)) / x)>N$ uniformly with respect to $k \in[0, T]$, where $N$ is the same as in $\left(\mathrm{H}_{5}\right)$.

Then the PBVP (1.1) has least two positive solutions.

Proof. Let $P$ be a cone in $E$ defined as in Theorem 3.2. From $\left(\mathrm{H}_{5}\right)$, there exist $\varepsilon>0$ and $r \in(0, e)$ such that

$$
f(k, x) \geq(N+\varepsilon) x, \quad k \in[0, T], x \in[0, r] .
$$

Then for each $x \in \partial P_{r}$, from (3.4) and the definition of $P$, we obtain

$$
\begin{aligned}
(\Phi x)(k) & =\sum_{s=0}^{T} G(k, s)[(M-1) x(s+1)+f(s, x(s+1))] \\
& \geq \sum_{s=0}^{T} G(k, s)[(M-1) x(s+1)+(N+\varepsilon) x(s+1)] \\
& >\sum_{s=0}^{T} G(k, s)(M-1+N) \delta\|x\| \\
& =\|x\| .
\end{aligned}
$$


Thus,

$$
\|\Phi x\|>\|x\| \quad \text { for } x \in \partial P_{r} .
$$

On the other hand, for each $x \in \partial P_{e}$, from $\left(\mathrm{H}_{6}\right)$ we obtain

$$
\begin{aligned}
(\Phi x)(k) & =\sum_{s=0}^{T} G(k, s)[(M-1) x(s+1)+f(s, x(s+1))] \\
& \leq \sum_{s=0}^{T} G(k, s)[(M-1) e+h(s)] \\
& <e=\|x\| .
\end{aligned}
$$

Hence,

$$
\|\Phi x\|<\|x\| \quad \text { for } x \in \partial P_{e} .
$$

In addition, from $\left(\mathrm{H}_{7}\right)$ there exist $\varepsilon^{\prime}>0$ and $e^{\prime}>e$ such that

$$
f(k, x) \geq\left(N+\varepsilon^{\prime}\right) x, \quad k \in[0, T], x \geq e^{\prime} .
$$

Choose $e^{*}=(1 / \delta) e^{\prime}$. Then for each $x \in \partial P_{e^{*}}$, we get $x(k) \geq \delta\|x\|=\delta e^{*}=e^{\prime}$.

Similar to (3.9), we have $\|\Phi x\|>\|x\|$ for $x \in \partial P_{e^{*}}$.

This, together with (3.10), (3.11) and Theorem 2.2, leads to the conclusion that there exist two positive solutions of (1.1): $x_{1} \in P_{e} \backslash \overline{P_{r}}$ and $x_{2} \in P_{e^{*}} \backslash \overline{P_{e}}$.

Corollary 3.1. Assume that $\left(\mathrm{H}_{1}\right)$, $\left(\mathrm{H}_{6}\right)$, and either $\left(\mathrm{H}_{5}\right)$ or $\left(\mathrm{H}_{7}\right)$ hold. Then the PBVP (1.1) has least one positive solution.

\section{Examples}

EXAMPle 4.1. Consider the PBVP

$$
\left\{\begin{array}{l}
\triangle x(k)=f(k, x(k+1)), \quad k \in[0,3], \\
x(0)=x(4)
\end{array}\right.
$$

where $T=3$ and

$$
f(k, x)= \begin{cases}-x+\frac{1}{6} k \ln (1+x), & k \in[0,3], x \in[0,1], \\ \frac{1}{2}(x-3)+\frac{1}{6} k \ln (1+x), & k \in[0,3], x \in[1,3], \\ \frac{1}{6} k \ln (1+x), & k \in[0,3], x \in[3,50], \\ (50-x)+\frac{1}{6} k \ln (1+x), & k \in[0,3], x \in[50,+\infty) .\end{cases}
$$

Then (4.1) has at least three nonnegative solutions. 
Proof. Choose $M=2, a=3, b=50$. Hence $b=50>48=3 \cdot 2^{4}=a M^{(T+1)}$. Then it is not difficult to show that $\left(\mathrm{H}_{1}\right)-\left(\mathrm{H}_{4}\right)$ hold. By Theorem 3.1, (4.1) has at least three nonnegative solutions.

EXAMPLE 4.2. Consider the PBVP

$$
\left\{\begin{array}{l}
\triangle x(k)=\frac{1+k}{400}\left[(x)^{(1 / 2)}+x^{2}\right]-x, \quad k \in[0,3], \\
x(0)=x(4),
\end{array}\right.
$$

Then (4.2) has at least two positive solutions.

Proof. Let $T=3$ and $f(k, x)=((1+k) / 400)\left[(x)^{(1 / 2)}+x^{2}\right]-x$.

Choose $M=2$; then $\delta=M^{-(T+1)}=2^{-4}=1 / 16$, and $\left(\mathrm{H}_{1}\right)$ is satisfied. Moreover, it is easy to see that

$$
\lim _{x \rightarrow 0+}((f(k, x)) / x)=+\infty \quad \text { and } \quad \lim _{x \rightarrow+\infty}((f(k, x)) / x)=+\infty
$$

both uniformly with respect to $k \in[0,3]$, that is, $\left(\mathrm{H}_{5}\right)$ and $\left(\mathrm{H}_{7}\right)$ are satisfied.

Now we prove that $\left(\mathrm{H}_{6}\right)$ is satisfied. Choose $e=16$; then $[\delta e, e]=[1,16]$. Let $h(k) \equiv-(49 / 50)$ for $k \in[0,3]$; then

$$
\begin{aligned}
f(k, x) & =\frac{1+k}{400}\left[(x)^{(1 / 2)}+x^{2}\right]-x \leq \frac{1}{100}\left[(x)^{(1 / 2)}+x^{2}\right]-x \\
& \leq-\frac{49}{50}, \quad k \in[0,3], \quad x \in[1,16] .
\end{aligned}
$$

So, $f(k, x) \leq h(k)<0, k \in[0,3], x \in[1,16]$, that is, $\left(\mathrm{H}_{6}\right)$ is satisfied. It follows from Theorem 3.3 that (4.2) has two positive solutions.

\section{References}

[1] R. P. Agarwal, Difference Equations and Inequalities (Marcel Dekker, New York, 1992).

[2] R. P. Agarwal, M. Bohner and P. J. Y. Wong, 'Eigenvalues and eigenfunctions of discrete conjugate boundary value problems', Comput. Math. Appl. 38(3-4) (1999), 159-183.

[3] R. P. Agarwal and J. Henderson, 'Positive solutions and nonlinear eigenvalue problems for thirdorder difference equations', Comput. Math. Appl. 36(10-12) (1998), 347-355.

[4] R. P. Agarwal and D. O'Regan, 'Multiple solutions for higher-order difference equations', Comput. Math. Appl. 37 (1999), 39-48.

[5] R. P. Agarwal and F. H. Wong, 'Existence of positive solutions for higher order difference equations', Appl. Math. Lett. 10(5) (1997), 67-74.

[6] R. P. Agarwal and P. J. Y. Wong, Advanced Topics in Difference Equations (Kluwer Academic, Dordrecht, 1997).

[7] D. R. Anderson, 'Discrete third-order three-point right-focal boundary value problems', Comput. Math. Appl. 45 (2003), 861-871.

[8] D. R. Anderson and R. I. Avery, 'Multiple positive solutions to a third-order discrete focal boundary value problem', Comput. Math. Appl. 42 (2001), 333-340.

[9] F. M. Atici and A. Cabada, 'Existence and uniqueness results for discrete second-order periodic boundary value problems', Comput. Math. Appl. 45 (2003), 1417-1427. 
[10] F. M. Atici and G. Sh. Guseinov, 'Positive periodic solutions for nonlinear difference equations with periodic cofficients', J. Math. Anal. Appl. 232 (1999), 166-182.

[11] R. I. Avery, C. J. Chyan and J. Henderson, 'Twin solutions of boundary value problems for ordinary differential equations and finite difference equations', Comput. Math. Appl. 42 (2001), 695-704.

[12] C. J. Chyan, J. Henderson and H. C. Lo, 'Existence of triple solutions of discrete $(n, p)$ boundary value problems', Appl. Math. Lett. 14 (2001), 347-352.

[13] P. W. Eloe, 'A generalization of concavity for finite differences', J. Math. Anal. Appl. 36(10-12) (1998), 109-113.

[14] D. Guo and V. Lakshmikantham, Nonlinear Problems in Abstract Cones (Academic Press, New York, 1988).

[15] Z. M. He, 'On the existence of positive solutions of $p$-Laplacian difference equations', J. Comput. Appl. Math. 161 (2003), 193-201.

[16] X. M. He and W. G. Ge, 'Triple solutions for second-order three-point boundary value problems', J. Math. Anal. Appl. 268 (2002), 256-265.

[17] X. M. He, W. G. Ge and M. S. Peng, 'Multiple positive solutions for one-dimensional $p$-Laplacian boundary value problems', Appl. Math. Lett. 15 (2002), 937-943.

[18] Z. M. He and J. S. Yu, 'On the existence of positive solutions of fourth-order difference equations', Appl. Math. Comput. 161 (2005), 139-148.

[19] J. Henderson, 'Positive solutions for nonlinear difference equations', Nonlinear Stud. 4(1) (1997), 29-36.

[20] J. Henderson and P. J. Y. Wong, 'Positive solutions for a system of nonpositive difference equations', Aequationes Math. 62 (2001), 249-261.

[21] W. G. Kelley and A. C. Peterson, Difference Equations (Academic Press, Boston, 1991).

[22] V. Lakshmikantham and D. Trigiante, Theory of Difference Equations: Numerical Methods and Applications (Academic Press, New York, 1988).

[23] R. W. Leggett and L. R. Williams, 'Multiple positive fixed points of nonlinear operators on ordered Banach spaces', Indiana Univ. Math. J. 28 (1979), 673-688.

[24] Y. S. Liu, 'Multiple solutions of periodic boundary value problems for first-order differential equations', Comput. Math. Appl. 54 (2007), 1-8.

[25] J. P. Sun, 'Positive solution for first-order discrete periodic boundary value problem', Appl. Math. Lett. 19 (2006), 1244-1248.

[26] J. P. Sun and W. T. Li, 'Multiple positive solutions of a discrete difference system', Appl. Math. Comput. 143 (2003), 213-221.

[27] Y. Wang and Y. M. Shi, 'Eigenvalues of second-order difference equations with periodic and antiperiodic boundary conditions', J. Math. Anal. Appl. 309 (2005), 56-69.

[28] P. J. Y. Wong, 'Positive solutions of difference equations with two-point right focal boundary conditions', J. Math. Anal. Appl. 224 (1998), 34-58.

[29] P. J. Y. Wong and R. P. Agarwal, 'Double positive solutions of $(n, p)$ boundary value problems for higher order difference equations', Comput. Math. Appl. 32 (1996), 1-21.

[30] _ 'Existence theorems for a system of difference equations with $(n, p)$-type conditions', Appl. Math. Comput. 123 (2001), 389-407.

[31] , 'Further results on fixed-sign solutions for a system of higher-order difference equations', Comput. Math. Appl. 42(3-5) (2001), 497-514.

DA-BIN WANG, Department of Applied Mathematics, Lanzhou University of Technology, Lanzhou, Gansu 730050, People's Republic of China e-mail: wangdb@lut.cn 